\title{
High-density lipoprotein subpopulation profiles in lipoprotein lipase and hepatic lipase deficiency
}

\author{
${ }^{1}$ Mariko Tani, ${ }^{1}$ Katalin V. Horvath, ${ }^{2}$ Benoit Lamarche, ${ }^{2}$ Patrick Couture, ${ }^{3}$ John R. Burnett, \\ ${ }^{1}$ Ernst J. Schaefer, ${ }^{1}$ Bela F. Asztalos*
}

\begin{abstract}
${ }^{1}$ Cardiovascular Nutrition Laboratory, Human Nutrition Research Center on Aging at Tufts University, Boston, MA, USA

${ }^{2}$ Institute on Nutraceuticals and Functional Foods, Laval University, Québec, Canada

${ }^{3}$ Department of Clinical Biochemistry, Path West Laboratory Medicine, Royal Perth and Fiona Stanley

Hospital Network and School of Medicine and Pharmacology, University of Western Australia, Perth, Australia
\end{abstract}
*Corresponding author:Cardiovascular Nutrition Laboratory, Jean Mayer USDA Human Nutrition, Research Center on Aging at Tufts University, 711 Washington Street, Boston, MA 02111.
Tel: (617) 556-3112; Fax:(617) 556-3103.
E-mail address: bela.asztalos@tufts.edu (B. F. Asztalos)

Key words: Apolipoproteins, Lipoprotein metabolism, HDL particles, HDL remodeling, Reverse cholesterol transport 


\section{Abstract:}

Background and aims: Our aim was to gain insight into the role that lipoprotein lipase (LPL) and hepatic lipase (HL) plays in HDL metabolism and to better understand LPL- and HL-deficiency states.

Methods: We examined the apolipoprotein (apo) A-I-, A-II-, A-IV-, C-I-, C-III-, and E-containing HDL subpopulation profiles, assessed by native 2-dimensional gel-electrophoresis and immunoblotting, in 6 homozygous and 11 heterozygous LPL-deficient, 6 homozygous and 4 heterozygous HL-deficient, and 50 control subjects.

Results: LPL-deficient homozygotes had marked hypertriglyceridemia and significant decreases in LDLC, HDL-C, and apoA-I. Their apoA-I-containing HDL subpopulation profile was shifted toward small HDL particles compared to controls. HL-deficient homozygotes had moderate hypertriglyceridemia, modest increases in LDL-C and HDL-C level, but normal apoA-I concentration. HL-deficient homozygotes had a unique distribution of apoA-I-containing HDL particles. The normally apoA-I:A-II, intermediate-size ( $\alpha-2$ and $\alpha-3)$ particles were significantly decreased, while the normally apoA-I only (very large $\alpha-1$, small $\alpha-4$, and very small pre $\beta-1$ ) particles were significantly elevated. In contrast to control subjects, the very large $\alpha-1$ particles of HL-deficient homozygotes were enriched in apoA-II. Homozygous LPL- and HL-deficient subjects also had abnormal distributions of apo C-I, C-III, and E in HDL particles. Values for all measured parameters in LPL- and HL-deficient heterozygotes were closer to values measured in controls than in homozygotes.

Conclusions: Our data are consistent with the concept that LPL is important for the maturation of small discoidal HDL particles into large spherical HDL particles, while HL is important for HDL remodeling of very large HDL particles into intermediate-size HDL particles.

Abbreviations: ABCA1, ATP-binding cassette transporter A1; apo, apolipoprotein; C, cholesterol; CE, cholesteryl ester; CETP, cholesteryl ester transfer protein; CHD, coronary heart disease; CVD, cardiovascular disease; FC, free cholesterol; HL, hepatic lipase; HDL, high-density lipoprotein; LCAT, lecithin:cholesterol acyltransferase; LDL, low-density lipoprotein; Lp lipoprotein; LPL, lipoprotein 
lipase; PL, phospholipid; RLP, remnant-like particle; SR-BI, scavenger receptor BI; TG, triglyceride; TRL, triglyceride-rich lipoprotein; VLDL, very-low-density lipoprotein. 


\section{Introduction:}

A low level of high-density lipoprotein cholesterol (HDL-C) is recognized as a major independent risk factor for the development of coronary heart disease (CHD) (1-3). HDL is a heterogeneous class of lipoprotein particles that differ in their protein and lipid composition and physiologic functions. Several enzymes play key roles in HDL remodeling in vivo including lipoprotein lipase (LPL) and hepatic lipase (HL).

LPL facilitates triglyceride (TG) lipolysis, while HL acts equally on TG and phospholipids. Both lipases reside mainly on the luminal surface of the capillary vascular endothelium. LPL hydrolyzes TG in triglyceride-rich lipoproteins (TRL) such as chylomicron and very-low density lipoprotein (VLDL), thus generating free fatty acids for uptake in adipose tissue as well as skeletal and cardiac muscle (4-6). TG hydrolysis by LPL reduces the core material of TRL, triggering release of surface components of these particles, namely apolipoproteins (except apoB), free cholesterol, and phospholipids, which are then transferred in part to HDL particles. It is well-documented that LPL deficiency causes very high levels of TRL and low levels of HDL-C (4-6).

$\mathrm{HL}$ is synthesized and secreted from hepatocytes and anchored to the liver sinusoidal surface by proteoglycans (7). HL hydrolyzes TG and phospholipids in apoB-containing lipoproteins and HDL, resulting in the formation of small HDL remnants (8). On the other hand, HL-deficient subjects have buoyant low-density lipoprotein (LDL) particles, increased cholesterol levels in $\mathrm{HDL}_{2}$, and $\mathrm{HDL}$ in these subjects is also enriched in TG (9-12). Hence, HL plays a role in determining the size, density, and metabolic fate of these lipoprotein particles by modulating their lipid composition (13). Ruel et al. have reported that HL-deficient homozygotes had substantial amounts of TG-enriched, large-size HDL particles (14). Three of those patients are also part of our present report.

In the past, our laboratory has characterized the apoA-I content of individual HDL particles as separated by 2-dimensional gel-electrophoresis of whole plasma followed by immunoblotting and image analysis. We have noted the consistent appearance of apoA-I in ten distinct HDL particles including the very small, discoidal pre $\beta$-1 HDL (5.6 nm), small, discoidal $\alpha-4$ HDL (7.4 nm), medium-size $\alpha-3$ HDL 
(8.1 nm), large $\alpha$-2 HDL $(9.2 \mathrm{~nm})$, and very large $\alpha-1$ HDL (11.0 nm) particles (15-19). We have also noted that apoA-II is found predominantly within medium-size $\alpha-3$ and large $\alpha-2$ HDL (apoA-I:A-II HDL particles), while none of the other HDL particles contain appreciable amounts of apoA-II, hence they have been denoted as apoA-I-only HDL particles (20). ApoE and apoA-IV are found on HDL particles that are each distinct from apoA-I-containing HDL particles (20). We have also documented that females have higher levels of apoA-I in very large $\alpha-1$ and large $\alpha-2$ HDL than males do (18). Both male and female patients with CHD, who are not on lipid-altering medications, have significantly lower levels of apoA-I in very large $\alpha-1$ HDL and significantly higher levels of apoA-I in very small pre $\beta-1$ HDL than healthy gender-matched control subjects do (15-19). Furthermore, we have documented that the concentrations of apoA-I in specific HDL particles are better markers of cardiovascular disease (CVD) risk than HDL-C is and that their levels predict progression or regression of angiographically-defined coronary artery disease (16-19). We have also characterized the specific and distinct apoA-I-containing HDL particle profiles of patients with deficiencies in apoA-I, ATP-binding cassette transporter A1 (ABCA1), lecithin:cholesterol acyltransferase (LCAT), and cholesteryl ester transfer protein (CETP), due to specific molecular defects (20-23).

In this study, we have now characterized the apoA-I, apoA-II, apoA-IV, apoC-I, apoC-III, and apoE content and distribution of HDL particles in homozygous and heterozygous LPL- and HL-deficient subjects and controls in order to better understand the roles of these two lipases in HDL particle metabolism.

\section{Materials and methods}

\section{Subjects}

Plasma samples from 5 homozygous, 1 compound heterozygous, and 4 heterozygous LPL-deficient patients were obtained. The patients were 4 males and 2 females, aged 23 to 43 years, with marked hypertriglyceridemia (> 1,000 mg/dL) and absent LPL activity in post-heparin plasma. They had no other diseases and were not on any medications that affect lipoprotein metabolism. The reported amino acid 
substitutions in homozygotes due to mutations in the LPL gene included: 1) Ala176 $\rightarrow$ Thr, 2)

Gly $188 \rightarrow$ Glu, 3) Ile194 $\rightarrow$ Thr, 4) Ile205 $\rightarrow$ Ser, 5) Pro207 $\rightarrow$ Leu, and 6) a compound heterozygote with a heterozygous Gly188 $\rightarrow$ Glu amino acid substitution and a nonsense mutation in exon 8 W394X $(24,25)$.

Plasma samples from 3 homozygous, 3 compound heterozygous, and 11 heterozygous HL-deficient patients were also obtained. HL homozygote-1 was a 56-year-old male noted to have significant hypertriglyceridemia, low post-heparin plasma LPL activity, and absent HL activity, with a homozygous intron-1 acceptor splice site mutation in the HL gene. He had sustained a myocardial infarction at age 41 years. HL homozygote-2 was a 53-year-old male with a major deletion involving the HL gene promoter and exon-1, modest hypertriglyceridemia, and absent post-heparin plasma HL activity and normal LPL activity off medications. He had sustained a myocardial infarction at age 37 years. HL homozygote-3 was a 64-year-old male with significant hypertriglyceridemia, low post-heparin LPL activity and absent HL activity, and a missense mutation Gly225 $\rightarrow$ Arg in HL. He had sustained a myocardial infarction at age 42 years. All of these patients had significant plasma TG lowering and normalized their post-heparin LPL activity on treatment with fibrate therapy (26). The three compound heterozygotes were a female and her two brothers with very low or undetectable post-heparin plasma HL activity due to a missense mutation in exon 5 Ala174 $\rightarrow$ Thr and a missense mutation Thr383 $\rightarrow$ Met in exon-8 of the HL gene (14). These latter patients also were noted to have moderate hypertriglyceridemia and marked TG enrichment of LDL and HDL particles.

Control subjects (25 males and 25 females) were selected from our previous studies based on having plasma samples available, no history of CVD or diabetes, and having a normal lipid profile (LDL-C $<130$ $\mathrm{mg} / \mathrm{dl}, \mathrm{HDL}-\mathrm{C}>40 \mathrm{mg} / \mathrm{dl}$ for males and $>50 \mathrm{mg} / \mathrm{dl}$ for female, and $\mathrm{TG}<150 \mathrm{mg} / \mathrm{dl}$ ).

Neither the patients nor the control subjects were on lipid-lowering medications at the time of sampling. All samples were frozen at $-80{ }^{\circ} \mathrm{C}$ and never thawed until just prior to the analysis described below. 


\section{Biochemical analyses}

Total-C, TG, HDL-C, and LDL-C concentrations were determined by standard enzymatic methods on an Olympus AU 400 analyzer using kits from Beckman-Coulter (Indianapolis, IN). ApoA-I concentrations were measured using a turbidimetric immunoassay (Wako Diagnostics, Richmond, VA). Within and between run coefficients of variation for all assays were $<5 \%$. Due to hyperlipidemia, the concentrations of less abundant apolipoproteins (A-II, A-IV, C-I, C-III, and E) could not be reliably measured from plasma; therefore we assessed only the distribution/co-migration of these apolipoproteins. Distributions of apo A-I, A-II, A-IV, C-I, C-III, and E in HDL particles were determined by nondenaturing 2-dimensional gel electrophoresis (2d-ndPAGE) of plasma followed by transferring HDL on nitrocellulose membrane and immunoblotting with specific antibodies, as previously described (15, 20). Briefly: 4 microliters of plasma were applied and electrophoresed on a vertical-slab agarose gel in the $1^{\text {st }}$ dimension. Gels were run in $4^{\circ} \mathrm{C}$ Tris-Tricine buffer at $250 \mathrm{~V}$ until the $\alpha$-mobility front moved $3.5 \mathrm{~cm}$ from the origin. The agarose gel was sliced, and the strips were applied onto 3-35\% non-denaturing concave gradient polyacrylamide gels. In the $2^{\text {nd }}$ dimension, gels were electrophoresed in $4^{\circ} \mathrm{C}$ TrisBorate buffer at $250 \mathrm{~V}$ for $24 \mathrm{~h}$, followed by electrotransfer to nitrocellulose membranes in $4^{\circ} \mathrm{C}$ TrisGlycine buffer at $30 \mathrm{~V}$ for $24 \mathrm{~h}$. The specific apolipoproteins were immuno-localized on the membrane with mono-specific goat anti-human primary antibodies against apolipoproteins A-I, A-II, A-IV, C-I, CIII, and E. ${ }^{125}$ I-labeled immunopurified goat-rabbit F(ab')2 IgG fraction (Thermo Fisher, cat\#A24449) as secondary antibody was then utilized. The bound ${ }^{125}$ I-labeled secondary antibody was quantified with a Storm 860 image analyzer (Molecular Dynamics, CA). Each membrane was first probed for the apolipoprotein of primary interest and then for apoA-I for reference. The sources of primary antibodies used in these experiments were: apo A-I, A-II, and A-IV antibodies prepared in our laboratory; apo C-I and C-III antibodies were provided by Dr. Alaupovic's laboratory as a gift; apoE antibody was purchased from Biodesign (cat\#K74190G). All antibodies were tested and validated for specificity by SDS-gel electrophoresis of normal human plasma followed by transferring the gel to nitrocellulose membrane and immunoblotting with the selected first antibodies and ${ }^{125}$ I-labeled secondary antibody. 


\section{Results}

\section{LPL deficiency}

In Table 1, we present the mean ( \pm SD) lipid and lipoprotein concentrations of 50 control, 6 homozygous and 4 heterozygous LPL-deficient subjects. Since the measured parameters in the compound heterozygote were not different from those in the homozygotes, we present all six subjects in the homozygote group. In the general population, HDL-related parameters are significantly different between males and females; however, this gender difference was not present in LPL-deficient homozygotes, therefore, we have not separated the data by gender. Compared to controls, complete lack of LPL activity was associated with about 21-fold higher than normal mean TG level and significantly lower LDL-C (-37\%), HDL-C (-75\%), and apoA-I (-67\%) levels. With regard to HDL particles, homozygotes had a mean increased concentration of apoA-I in pre $\beta-1$ HDL $(+35 \%)$, while apoA-I in other HDL particles decreased with increasing size: small $\alpha-4$ (-23\%), medium $\alpha-3$ (-61\%), large $\alpha-2$ ($74 \%)$, and very large $\alpha-1(-89 \%)$, as compared to controls. Decreases of apoA-I in the latter three HDL particles were significant $(\mathrm{p}<0.05)$. The apoA-I-containing HDL subpopulation profiles of a representative control, a heterozygous, and a homozygous LPL-deficient subject are shown in Figure 1. In Supplementary Figure 1, we present the apoA-I-containing HDL subpopulations of each homozygous LPL-deficient subjects, LPL-deficient (patient 6) being the compound heterozygous subject. A shift towards smaller particles, especially pre $\beta-1$, and lack of larger $\alpha$-mobility particles is visible in LPLdeficient homozygotes. The HDL subpopulation profile in LPL heterozygotes was slightly skewed toward small HDL particles, but the differences from controls did not reach significance.

The distributions of apoA-II, apoC-I, apoC-III and apoE in a representative control and a homozygous LPL-deficient subject are shown in Figure 2. The overall signal of apoA-II in homozygotes was low but the distribution and sizes of the apoA-II-containing HDL particles ( $\alpha-2$ and $\alpha-3)$ appeared to be normal. Most of apoC-I in controls was associated with very large $\alpha-1$ HDL particles, while in homozygotes, only trace amount of apoC-I were seen in HDL, due to a marked decrease of its normal 
carrier particle, $\alpha-1$ HDL. In controls, about $85 \%$ of apoC-III was found in various-sized $\alpha$-mobility HDL particles with a small amount of apoC-III being found in very small, lipid-poor, pre $\beta$-migrating HDL particles. In contrast, in LPL-deficient homozygotes, most of apoC-III was found in very small, lipidpoor, pre $\beta$-migrating HDL particles. In controls, a small amount of apoE was found in VLDL and the majority was in distinct very large (> 11-20 nm diameter) LpE particles with pre $\beta$ - or slow- $\alpha$-mobility. Most of the LpE particles were larger than $\alpha-1$ HDL. In LPL-deficient homozygotes, apoE was found in significantly smaller size HDL particles, but still with pre $\beta$ - or slow- $\alpha$-mobility. As in controls, these particles were separate from apoA-I-containing HDL particles. The distribution of apoA-IV in homozygotes was not different from those in controls (data not shown). No significant differences of any of the above-listed parameters were noted in LPL-deficient heterozygotes as compared to control subjects.

\section{HL deficiency}

In Table 1, we also present the mean $( \pm \mathrm{SD})$ lipid and lipoprotein concentrations of 6 homozygous and 11 heterozygous HL-deficient subjects, compared to 50 controls. Similar to LPL-deficient patients, we have not observed significant differences between HL homozygotes and compound heterozygotes or between genders. Therefore, we again present the pulled data for complete HL-deficient patients. HL homozygotes had significantly higher plasma TG (4-fold) and HDL-C levels (+31\%) than controls. They had normal plasma apoA-I level but apoA-I distribution in the HDL particles was different from normal. They had significantly increased level of apoA-I in very large $\alpha-1(+66 \%)$, small $\alpha-4(+65 \%)$, and verysmall pre $\beta-1$ HDL particles $(+215 \%)$, and significantly decreased level of apoA-I in large $\alpha-2(-53 \%)$ and medium-size $\alpha-3$ HDL particles (-44\%), compared to controls. HL heterozygotes had significantly increased total and LDL-C levels, but normal levels of HDL-C, apoA-I and HDL particles.

In Figure 1, we also present the apoA-I-containing HDL subpopulation profile of a representative heterozygous and homozygous HL-deficient subject. The apoA-I-containing HDL subpopulations of the three homozygous (patients 1-3) and the three compound heterozygous (patients 4-6) HL-deficient patients are presented in Supplementary Figure 2. The electrophoretograms show increased 
concentrations of apoA-I in the large $\alpha-1$ and small $\alpha-4$, and pre $\beta-1$ HDL particles as well as decreased concentrations of apoA-I in the intermediate sized $\alpha-2$ and $\alpha-3$.

In Figure 3, we present the distribution of apoA-II, apoC-I, apoC-III and apoE in a representative control and a representative homozygous HL-deficient subject. In controls, the majority of apoA-II was present in $\alpha-2$ and $\alpha-3$ HDL particles, while in HL-deficient homozygotes, all $\alpha$-mobility particles were highly enriched in apoA-II. The majority of apoC-I in controls was found in very large $\alpha-1$ HDL particles, while in HL homozygotes, the majority of apoC-I was present in very small particles, co-migrating with albumin. The pattern of apoC-III-containing HDL particles was similar between controls and HL homozygotes, but the distribution of apoC-III in homozygotes was markedly shifted towards very small, lipid-poor HDL particles with $\alpha$ - and pre $\beta$-migration. In contrast to controls, where the majority of apoE was found in $<17 \mathrm{~nm}$ HDL particles, the majority of apoE in homozygotes was associated with VLDL particles. The distribution of apoA-IV in HDL particles in homozygotes was not different from controls (data not shown). Similar to apoA-I, the distribution patterns of other HDL apolipoproteins (A-II, A-IV, C-I, C-III, and E) in HL-deficient heterozygotes were not significantly different from those in control subjects.

\section{Discussion}

Patients with homozygous LPL deficiency are known to have marked hypertriglyceridemia along with decreased levels of LDL-C and HDL-C and to be at increased risk of developing recurrent pancreatitis (4). Our LPL-deficient homozygotes had a mean TG level of $>2,000 \mathrm{mg} / \mathrm{dL}$ and decreased concentrations of LDL-C and HDL-C. The HDL subpopulation profile of these patients was markedly shifted toward small-size HDL particles. When chylomicrons and VLDL undergo lipolysis by LPL, the core of these particles shrinks and the surface components, including phospholipids, free cholesterol, and non-apoB apolipoproteins, are shed and provide components for the formation of larger HDL particles. Therefore, LPL is critical not only for the lipolysis and metabolism of TG-rich lipoproteins (TRL) but also for the normal transformation of small, lipid-poor, discoidal HDL particles into larger and more 
complex spherical HDL particles. As a consequence of LPL-mediated decrease of TRL, CETP activity decline. CETP activity correlates with the concentration of TRL, but not with the concentration of CETP protein (27). In case of LPL deficiency and high plasma TG level, CETP enriches large HDL particles with TG. As a result, HL activity increases on the TG-enriched HDL particles. As TG is hydrolyzed by HL the core of HDL particles shrink and the extra surface material (free fatty acids, unesterified cholesterol, phospholipids, and apolipoproteins) sheds and the particles' size decrease. ApoA-II, apoC-I, and apoC-III are decreased along with apoA-I especially in the large HDL particles indicating that apoAI-containing HDL particles are essential carriers for those apolipoproteins. The significant shift of apoEcontaining particles toward smaller size indicates that LPL activity is also essential for the maturation of those particles.

Our homozygous HL-deficient patients had levels of TG, LDL-C, and HDL-C that were about 4-fold, 1.3-fold, and 1.3-fold higher than control values, respectively, and all had premature CVD. Though homozygotes had significantly elevated HDL-C level, their apoA-I concentration was not different from control values, however the distribution of apoA-I within HDL particles was different and unique for this condition. The normally apoA-I-only very large $\alpha-1$ particles were significantly elevated and enriched in apoA-II, while the normally apoA-I:A-II, intermediate-size, $\alpha-2$ and $\alpha-3$ particles were significantly decreased in HL-deficient homozygotes. In these patients, the small apoA-I-only particles, $\alpha-4$ and pre $\beta$ 1, were also elevated. Under normal conditions, HL works in concert with LPL and CETP and it is hard to figure out its role in HDL particle metabolism. In HL-deficient homozygotes, we were able to dissect some of the individual roles of HL on the distribution and composition of HDL particles. HL deficiency not only causes changes in HDL's lipid composition but also in the redistribution of all major apolipoproteins. Moreover, we have learned that HL activity is essential for the formation of distinct LpA-I ( $\alpha-1, \alpha-4$ and pre $\beta-1)$ and LpA-I:A-II ( $\alpha-2$ and $\alpha-3)$ HDL particles as well as for the formation of large, lipidated apoE-containing HDL particles.

HL is not the only player in the catabolism of large HDL particles. Recently it has been documented that endothelial lipase (EL) deficiency is also associated with increased levels of large-size HDL particles, 
although the HDL-particle profile was different from that observed in HL deficiency (28). HL has primarily triglyceride lipase activity and acts on all lipoprotein classes, while EL hydrolyzes phospholipids primarily on HDL. In contrast to these lipases, we have not found significantly altered HDL subpopulation profile in patients with lysosomal acid-lipase (LAL) deficiency despite having significantly decreased HDL-C levels (29). It is worth noting that so far, neither EL- nor LAL-deficiency has been found to be significantly associated with CVD risk.

Previously we have characterized the specific and distinct apoA-I-containing HDL particle profiles of patients with deficiencies in apoA-I, ABCA1, LCAT, and CETP, due to specific molecular defects (2023). We have shown that apoA-I secretion, ABCA1-dependent cell-cholesterol efflux, and cholesterol esterification via LCAT are all critical for the formation and maturation of small, discoidal particles into larger and increasingly spherical HDL particles. Patients with complete apoA-I deficiency had HDL-C < $5 \mathrm{mg} / \mathrm{dL}$ and no apoA-I in HDL particles (21). Patients with Tangier disease (homozygous ABCA1 deficiency) had HDL-C levels $<10 \mathrm{mg} / \mathrm{dL}$ and apoA-I only in very small, discoidal pre $\beta-1$ HDL particles (22). Homozygous LCAT-deficient patients had HDL-C levels $<15 \mathrm{mg} / \mathrm{dL}$ and only discoidal HDL particles, mainly small pre $\beta-1$ and $\alpha-4$ particles, containing two apoA-I molecules and in some extent larger $(8-17 \mathrm{~nm})$ discs containing multiple apoA-I molecules (20). We have also shown that lipid-poor, discoidal pre $\beta-1$ particles mature into large spherical HDL particles (30) and that exposure of large HDL particles to scavenger receptor BI (SR-BI) results in the disintegration of these large particles into smaller, $\alpha-3$ and $\alpha-4$, HDL particles (31). Subjects with CETP deficiency had markedly increased HDL-C levels and abnormally large HDL particles, which besides apoA-I also contained apoA-II and apoE, and markedly decreased concentrations of all normal-size $\alpha$-mobility $(\alpha-1, \alpha-2, \alpha-3$, and $\alpha-4)$ HDL particles (23). In normal subjects, in the presence of CETP, these abnormally large HDL particles are rapidly converted into distinct LpE- and a step smaller LpA-I:A-II HDL particles. The fractional catabolic rate (FCR) of apoA-I has a strong positive association with plasma TG level and an inverse association with HDL particle size $(32,33)$. HL-deficient patients have mainly large TG-enriched HDL particles (14) and decreased apoA-I FCR (34). 
Based on our previous and present work, we have updated our model of HDL-particle metabolism and remodeling as shown in Figure 4. HDL particle formation and maturation (green lines): 1) ApoA-I is synthesized in the liver and the small intestine and is secreted in the form of monomeric apoA-I molecules, which rapidly acquire some phospholipids in the circulation. The small amount of phospholipid triggers the monomeric apoA-I molecules to dimerize and form very small discoidal pre $\beta-1$ HDL particles (22). 2) When pre $\beta-1$ particles gain cholesterol via ABCA1, the majority of pre $\beta-1$ particles are transformed into small discoidal $\alpha-4$ particles containing two molecules of apoA-I. The minority of pre $\beta-1$ particles form larger particles containing 3-5 apoA-I and only small amount of lipids, therefore these particles are large discs (apoA-I aggregates). Pre $\beta-1$ particles gain cellular cholesterol via ABCA1 and are rapidly transformed into discoidal $\alpha$-mobility HDL particles containing two molecules of apoA-I ( $\alpha-4)$ and -to a lesser extent- larger discs containing 3-5 apoA-I molecules. Under normal condition, the larger multi-apoA-I-containing discs are transient with very short half-life time; however they can be detected in LCAT-deficient subjects (20). 3) LCAT esterifies free cholesterol preferentially in larger discs. 4) The increased lipid content and the presence of cholesteryl esters in the core facilitate apoA-II binding to these particles. 5) Through the action of LPL, lipids and apolipoproteins are released from TRL particles and become available for small HDL particles to mature in size and complexity. 6) In response to changes in the core and surface components, particles fuse into abnormally large (12-17 nm), undifferentiated (LpA-I:A-II:E) HDL particles. Again, under normal conditions, the half-life time of these particles are very short; however they can be seen in CETP-deficient subjects (23).

HDL particle remodeling (brown lines): 7) CETP exchanges cholesteryl ester for TG between HDL and TRL particles and transforms LpA-I:A-II:E HDL into distinct LpA-I:A-II and LpE HDL particles. 8) HL hydrolyzes TG and as the core shrinks the very large LpA-I:A-II HDLs split into particles containing only apoA-I (small pre $\beta-1$ and large $\alpha-1$ HDL) and particles containing both apoA-I and apoA-II (medium-size $\alpha-3$ ). 9) The cholesterol-rich $\alpha-1$ and $\alpha-2$ HDL particles interact with SR-BI, which selectively takes up cholesterol from these particles. The remaining cholesterol-depleted particles return into the circulation as $\alpha-3$ and $\alpha-4$ particles (31). The end products of HDL remodeling and degradation 
are small $\alpha-4$ and very small pre $\beta-1$ HDL particles, which are either reutilized to form larger HDL particles or catabolized: 10) Pre $\beta-1$ HDL particles are removed from plasma by the kidney and $\alpha-4$ HDL by the liver (blue lines).

HDL-particle formation has recently been described based on stable-isotope-labeled apoA-I kinetics in HDL particles (35). Mendivil et al. suggest that multiple-size complex HDL particles are simultaneously secreted from cells and there is no precursor-product relationship between pre $\beta-1$ and larger HDL particles. In contrast, we believe that cells secrete monomer apoA-I and that spontaneously picks up some phospholipid and forms pre $\beta-1$ (a poorly-lipidated apoA-I dimer) in plasma. Multiple apoA-I-containing (multiple size) HDL discs form after pre $\beta$-1 HDL particles efflux lipids from cells (step 2). This concept is supported by Dr. Phillips' data showing that larger multiple apoA-I-containing discs form from lipid-free apoA-I and the size of the discs depends on the apoA-I/cholesterol ratio (36, 37). Our model of HDL particle metabolism is also in line with Dr. Zannis' model, which indicates that pre $\beta-1$ is the precursor of larger HDL particles (38). Moreover, we have previously reported that when partially delipidated monkey HDL (containing about $60 \%$ of apoA-I in small pre $\beta$ - and $\alpha$-mobility particles) was re-infused into the same animals small HDL particles were converted entirely into larger $\alpha$ HDL particles indicating that small HDL particles were precursors of larger HDL particles (30).

Normally, HDL remodeling is a rapid process and many of the intermediate particles have very short half-life time. However, we can observe and measure these transient particles in patients with errors in the process of HDL metabolism/remodeling, which substantially slow the conversion of these particles. Therefore, we believe that the data we have generated on the metabolism/remodeling of HDL particles in patients with inborn errors in the process are important to complement data generated by stable-isotope labeled apoA-I in normal people.

Complete deficiency of LPL or HL results in very distinct HDL subpopulation profiles. Our data are consistent with the concept that LPL is important for the maturation of small discoidal HDL particles into large spherical HDL particles, while HL is important for HDL remodeling of very large HDL particles 
into intermediate-size HDL particles. These data also support our hypothesis that HDL metabolism is not a simple linear size-expansion of HDL particles.

\section{Financial support}

This research was supported by grant (HL117933) from the National Heart, Lung, and Blood Institute to BFA and by contract (53-3K06-5-10) from the US Department of Agriculture to EJS. JRB was supported by a Practitioner Fellowship from the Royal Perth Hospital Medical Research Foundation.

\section{Dedication}

Dr. John Brunzell, who gave us samples from many of the lipase-deficient patients and initially participated in the writing of this manuscript, recently passed away at the age of 78 years after a long and distinguished career at the University of Washington, Seattle. He made many contributions to our field, and had a long and abiding interest in the effects of lipoprotein and hepatic lipases on human lipoprotein metabolism. We dedicate this paper to his memory. He will be greatly missed by many investigators in the field. 


\section{References}

1. Gordon DJ, Probstfield JL, Garrison RJ, Neaton JD, Castelli WP, Knoke JD, Jacobs DR Jr, Bangdiwala S, Tyroler HA. High-density lipoprotein cholesterol and cardiovascular disease. Four prospective American studies. Circulation. 1989;79(1):8-15.

2. Ballantyne CM, Herd JA, Ferlic LL, Dunn JK, Farmer JA, Jones PH, Schein JR, Gotto AM Jr. Influence of low HDL on progression of coronary artery disease and response to fluvastatin therapy. Circulation. 1999;99(6):736-43.

3. Nicholls SJ, Tuzcu EM, Sipahi I, Grasso AW, Schoenhagen P, Hu T, Wolski K, Crowe T, Desai MY, Hazen SL, Kapadia SR, Nissen SE. Statins, high-density lipoprotein cholesterol, and regression of coronary atherosclerosis. JAMA. 2007;297(5):499-508.

4. Brunzell J. Familial lipoprotein lipase deficiency and other causes of the chylomicronemia syndrome. In: Sriver C, Baudet A, Sly w, Valle D. eds. The Metabolic and Molecular Basis of Inherited Disease. $7^{\text {th }}$ ed. New York: 1995:1913-32

5. Zheng C, Murdoch SJ, Brunzell JD, Sacks FM. Lipoprotein lipase bound to apolipoprotein B lipoproteins accelerates clearance of postprandial lipoproteins in humans. Arterioscler Thromb Vasc Biol. 2006;26(4):891-6.

6. Beisiegel U, Weber W, Bengtsson-Olivecrona G. Lipoprotein lipase enhances the binding of chylomicrons to low density lipoprotein receptor-related protein. Proc Natl Acad Sci USA. $1991 ; 88(19): 8342-6$.

7. Doolittle MH, Wong H, Davis RC, Schotz MC. Synthesis of hepatic lipase in liver and extrahepatic tissues. J Lipid Res. 1987;28(11):1326-34.

8. Kuusi T, Saarinen P, Nikkilä EA. Evidence for the role of hepatic endothelial lipase in the metabolism of plasma high density lipoprotein2 in man. Atherosclerosis. 1980;36(4):589-93.

9. Hegele RA, Little JA, Vezina C, Maguire GF, Tu L, Wolever TS, Jenkins DJ, Connelly PW. Hepatic lipase deficiency. Clinical, biochemical, and molecular genetic characteristics. Arterioscler Thromb. $1993 ; 13(5): 720-8$. 
10. Connelly PW, Maguire GF, Lee M, Little JA. Plasma lipoproteins in familial hepatic lipase deficiency. Arteriosclerosis. 1990;10(1):40-8.

11. Breckenridge WC, Little JA, Alaupovic P, Wang CS, Kuksis A, Kakis G, Lindgren F, Gardiner G. Lipoprotein abnormalities associated with a familial deficiency of hepatic lipase. Atherosclerosis. 1982;45(2):161-79.

12. Carlson LA, Holmquist L, Nilsson-Ehle P. Deficiency of hepatic lipase activity in post-heparin plasma in familial hyper-alpha-triglyceridemia. Acta Med Scand. 1986;219(5):435-47.

13. Rubinstein A, Gibson JC, Paterniti, Jr. JR, G. Kakis G, Little A, Ginsberg HN, Brown WV. Effect of heparin-induced lipolysis on the distribution of apolipoprotein E among lipoprotein subclasses. Studies with patients deficient in hepatic triglyceride lipase and lipoprotein lipase. J. Clin. Invest. 1985;75: 710721.

14. Ruel IL, Couture P, Gagne C, Deshaies Y, Simard J, Hegele RA, Lamarche B. Characterization of a novel mutation causing hepatic lipase deficiency among French Canadians. J Lipid Res. 2003;44(8):1508-14.

15. Asztalos BF, Roheim PS, Milani RL, Lefevre M, McNamara JR, Horvath KV, Schaefer EJ. Distribution of ApoA-I-containing HDL subpopulations in patients with coronary heart disease. Arterioscler Thromb Vasc Biol. 2000;20(12):2670-6.

16. Asztalos BF, Cupples LA, Demissie S, Horvath KV, Cox CE, Batista MC, Schaefer EJ. High-density lipoprotein subpopulation profile and coronary heart disease prevalence in male participants of the Framingham Offspring Study. Arterioscler Thromb Vasc Biol. 2004;24:2181-2187.

17. Asztalos BF, Collins D, Cupples LA, Demissie S, Horvath KV, Bloomfield HE, Robins SJ, Schaefer EJ. Value of high-density lipoprotein (HDL) subpopulations in predicting recurrent cardiovascular events in the Veterans Affairs HDL Intervention Trial. Arterioscler Thromb Vasc Biol. 2005;25(10):2185-91.

18. Lamon-Fava S, Herrington DM, Reboussin DM, Sherman M, Horvath KV, Cupples LA, White C, Demissie S, Schaefer EJ, Asztalos BF. Plasma levels of HDL subpopulations and remnant lipoproteins predict the extent of angiographically defined disease in post-menopausal women. Arterioscler Thromb Vasc Biol 2008; 28:575-579. 
19. Asztalos BF, Batista M, Horvath KV, Cox CE, Dallal GE, Morse JS, Brown GB, Schaefer EJ. Change in alpha1 HDL concentration predicts progression in coronary artery stenosis. Arterioscler Thromb Vasc Biol. 2003;23(5):847-52.

20. Asztalos BF, Schaefer EJ, Horvath KV, Yamashita S, Miller M, Franceschini G, Calabresi L. Role of LCAT in HDL remodeling: investigation of LCAT deficiency states. J Lipid Res. 2007;48(3):592-9.

21. Santos RD, Schaefer EJ, Asztalos BF, Polisecki E, Wang J, Hegele RA, Martinez LR, Miname MH, Rochitte CE, Da Luz PL, Maranhão RC. Characterization of high density lipoprotein particles in familial apolipoprotein A-I deficiency. J Lipid Res. 2008;49(2):349-57.

22. Asztalos BF, Brousseau ME, McNamara JR, Horvath KV, Roheim PS, Schaefer EJ. Subpopulations of high density lipoproteins in homozygous and heterozygous Tangier disease. Atherosclerosis. 2001;156(1):217-25.

23. Asztalos BF, Horvath KV, Kajinami K, Nartsupha C, Cox CE, Batista M, Schaefer EJ, Inazu A, Mabuchi H. Apolipoprotein composition of HDL in cholesteryl ester transfer protein deficiency. J Lipid Res. 2004;45(3):448-55.

24. Peterson J, Ayyobi AF, Ma Y, Henderson H, Reina M, Deeb SS, Santamarina-Fojo S, Hayden MR, Brunzell JD. Structural and functional consequences of missense mutations in exon 5 of the lipoprotein lipase gene. J Lipid Res. 2002;43(3):398-406.

25. Hooper AJ, Crawford GM, Brisbane JM, Robertson K, Watts GF, van Bockxmeer FM, Burnett JR. Familial lipoprotein lipase deficiency caused by known (G188E) and novel (W394X) LPL gene mutations. Ann Clin Biochem. 2008;45:102-5.

26. Zambon A, Deeb SS, Bensadoun A, Foster KE, Brunzell JD. In vivo evidence of a role for hepatic lipase in human apoB-containing lipoprotein metabolism, independent of its lipolytic activity. J Lipid Res. 2000;41(12):2094-99.

27. Asztalos BF, Swarbrick MM, Schaefer EJ, Dallal GE, Horvath KV, Ai M, Stanhope KL, AustrheimSmith I, Wolfe BM, Ali M, Havel PJ. Effects of weight loss, induced by gastric bypass surgery, on HDL remodeling in obese women. J Lipid Res. 2010 Aug;51(8):2405-12. 
28. Singaraja RR, Sivapalaratnam S, Hovingh K, Dubé MP, Castro-Perez J, Collins HL, Adelman SJ, Riwanto M, Manz J, Hubbard B, Tietjen I, Wong K, Mitnaul LJ, van Heek M, Lin L, Roddy TA, McEwen J, Dallinge-Thie G, van Vark-van der Zee L, Verwoert G, Winther M, van Duijn C, Hofman A, Trip MD, Marais AD, Asztalos B, Landmesser U, Sijbrands E, Kastelein JJ, Hayden MR. The impact of partial and complete loss-of-function mutations in endothelial lipase on high-density lipoprotein levels and functionality in humans. Circ Cardiovasc Genet. 2013;6(1):54-62.

29. Pullinger CR, Stock EO, Movsesyan I, Malloy MJ, Frost PH, Tripuraneni R, Quinn AG, Ishida BY, Schaefer EJ, Asztalos BF, Kane JP. Identification and metabolic profiling of patients with lysosomal acid lipase deficiency. J Clin Lipidol. 2015;9(5):716-26.

30. Sacks FM, Rudel LL, Conner A, Akeefe H, Kostner G, Baki T, Rothblat G, de la Llera-Moya M, Asztalos B, Perlman T, Zheng C, Alaupovic P, Maltais JA, Brewer HB. Selective delipidation of plasma HDL enhances reverse cholesterol transport in vivo. J Lipid Res. 2009;50(5):894-907.

31. Webb NR, de Beer MC, Asztalos BF, Whitaker N, van der Westhuyzen DR, de Beer FC. Remodeling of HDL remnants generated by scavenger receptor class B type I. J Lipid Res. 2004;45(9):1666-73.

32. Schaefer EJ, Zech LA, Jenkins LL, Bronzert TJ, Rubalcaba EA, Lindgren FT, Aamodt RL, Brewer HB Jr. Human apolipoprotein A-I and A-II metabolism. J Lipid Res. 1982;23(6):850-62.

33. Brinton EA, Eisenberg S, Breslow JL. Human HDL cholesterol levels are determined by apoA-I fractional catabolic rate, which correlates inversely with estimates of HDL particle size. Effects of gender, hepatic and lipoprotein lipases, triglyceride and insulin levels, and body fat distribution. Arterioscler Thromb. 1994;14(5):707-20.

34. Ruel IL, Couture P, Cohn JS, Bensadoun A, Marcil M, Lamarche B. Evidence that hepatic lipase deficiency in humans is not associated with pro atherogenic changes in HDL composition and metabolism. J Lipid Res. 2004;45(8):1528-37.

35. Mendivil CO, Furtado J, Morton AM, Wang L, Sacks FM. Novel Pathways of Apolipoprotein A-I Metabolism in High-Density Lipoprotein of Different Sizes in Humans. Arterioscler Thromb Vasc Biol. 2016;36(1):156-65. 
36. Duong PT, Collins HL, Nickel M, Lund-Katz S, Rothblat GH, Phillips MC. Characterization of nascent HDL particles and microparticles formed by ABCA1-mediated efflux of cellular lipids to apoA-I. J Lipid Res. 2006;47(4):832-43.

37. Lyssenko NN, Nickel M, Tang C, Phillips MC. Factors controlling nascent high-density lipoprotein particle heterogeneity: ATP-binding cassette transporter A1 activity and cell lipid and apolipoprotein AI availability. FASEB J. 2013;27(7):2880-92.

38. Zannis VI, Fotakis P, Koukos G, Kardassis D, Ehnholm C, Jauhiainen M, Chroni A. HDL Biogenesis, Remodeling, and Catabolism in High Density Lipoproteins, Handbook of Experimental Pharmacology 224, Springer, Editors Arnold von Eckardstein, Dimitris Kardassis. 2015; 53-113. 
Table 1. Lipoprotein parameters of study participants.

\begin{tabular}{|c|c|c|c|c|c|}
\hline & \multirow[t]{2}{*}{ Control } & \multicolumn{2}{|c|}{ LPL-deficient } & \multicolumn{2}{|c|}{ HL-deficient } \\
\hline & & Homozygote & Heterozygote & Homozygote & Heterozygote \\
\hline & $(\mathrm{n}=50)$ & $(\mathrm{n}=6)$ & $(\mathrm{n}=4)$ & $(n=6)$ & $(n=11)$ \\
\hline TC & $178 \pm 27$ & $311 \pm 72 *$ & $175 \pm 14$ & $265 \pm 41^{*}$ & $212 \pm 30^{*}$ \\
\hline LDL-C & $99 \pm 21$ & $62 \pm 21 *$ & $125 \pm 9$ & $135 \pm 37$ & $155 \pm 17 *$ \\
\hline TG & $111 \pm 36$ & $2330 \pm 488^{*}$ & $145 \pm 24$ & $461 \pm 256^{*}$ & $121 \pm 31$ \\
\hline HDL-C & $59 \pm 10$ & $15 \pm 8^{*}$ & $45 \pm 11$ & $77 \pm 14^{*}$ & $65 \pm 12$ \\
\hline ApoA-I & $170 \pm 22$ & $56 \pm 9 *$ & $152 \pm 12$ & $175 \pm 18$ & $165 \pm 11$ \\
\hline \multicolumn{6}{|c|}{ Major HDL particles (expressed as mg apoA-I) } \\
\hline Pre $\beta-1$ & $8.4 \pm 3.2$ & $11.3 \pm 9.5$ & $10.1 \pm 3.0$ & $18.1 \pm 6.1^{*}$ & $9.1 \pm 3.7$ \\
\hline$\alpha-1$ & $35.7 \pm 13.1$ & $3.8 \pm 1.6^{*}$ & $27.8 \pm 8.8$ & $59.3 \pm 13.5^{*}$ & $34.6 \pm 10.3$ \\
\hline$\alpha-2$ & $66.1 \pm 11.6$ & $17.3 \pm 3.5^{*}$ & $61.0 \pm 12.2$ & $31.0 \pm 8.1^{*}$ & $65.9 \pm 9.9$ \\
\hline$\alpha-3$ & $21.5 \pm 4.0$ & $8.3 \pm 1.9^{*}$ & $20.1 \pm 3.9$ & $12.1 \pm 3.7^{*}$ & $23.1 \pm 4.5$ \\
\hline$\alpha-4$ & $14.7 \pm 3.9$ & $11.3 \pm 1.5$ & $16.7 \pm 3.6$ & $24.2 \pm 5.1 *$ & $16.6 \pm 3.9$ \\
\hline
\end{tabular}

Numbers represent mean $(\mathrm{mg} / \mathrm{dL}) \pm \mathrm{SD} ;{ }^{*} p<0.05$ compared to control subjects.

$1 \mathrm{mg} / \mathrm{dL}$ cholesterol $=0.02586 \mathrm{mmol} / \mathrm{L} ; 1 \mathrm{mg} / \mathrm{dL} \mathrm{TG}=0.01129 \mathrm{mmol} / \mathrm{L}$. 


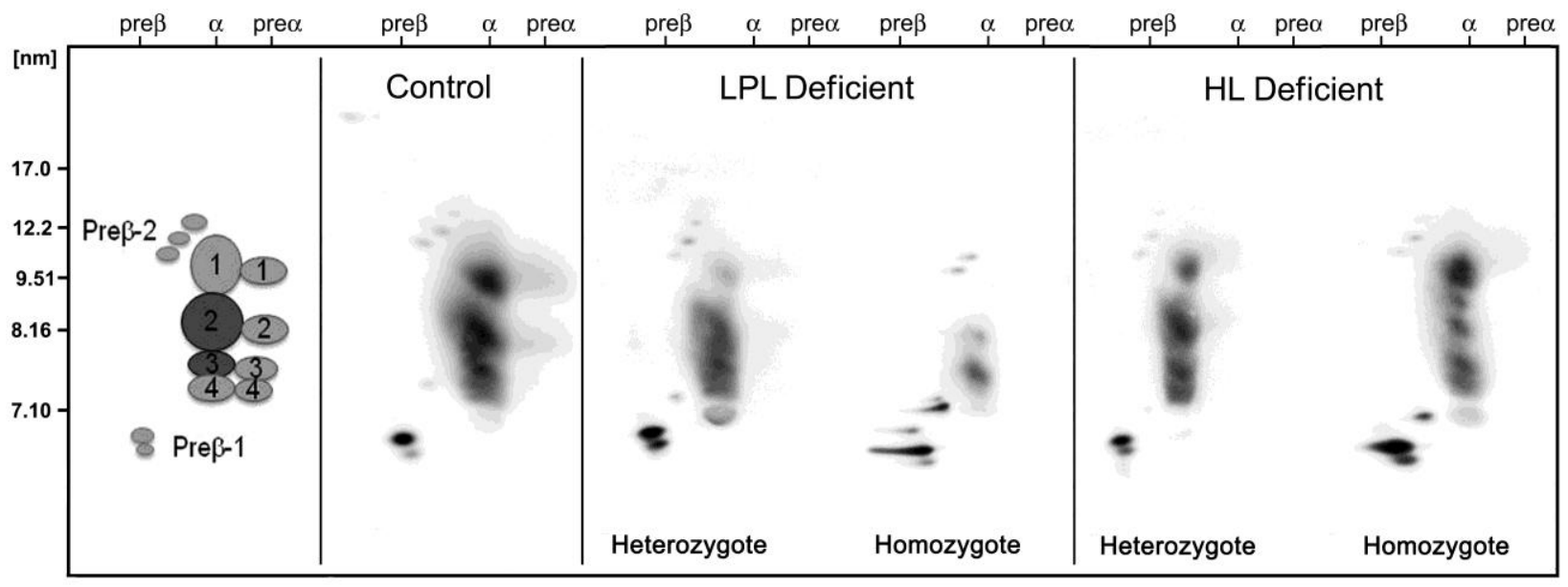

Fig. 1: ApoA-I-containing HDL subpopulations of a representative control (LDL-C=105 mg/dL, TG 87 mg/dL, HDL-C 54 mg/dL, apoA-I 165 mg/dL, and no history of CVD), a heterozygous LPL-deficient (LDL-C=120 mg/dL, TG 137 mg/dL, HDL-C 40 mg/dL, apoA-I 145 mg/dL), a homozygous LPLdeficient (LDL-C=65 mg/dL, TG 2740 mg/dL, HDL-C 14 mg/dL, apoA-I 55 mg/dL), a heterozygous HL-deficient (LDL-C=140 mg/dL, TG $120 \mathrm{mg} / \mathrm{dL}$, HDL-C 74 mg/dL, apoA-I $175 \mathrm{mg} / \mathrm{dL}$ ), and a homozygous HL-deficient (LDL-C=122 mg/dL, TG 500 mg/dL, HDL-C 64 mg/dL, apoA-I 161 mg/dL) subject. HDL particles were separated from plasma by non-denaturing $2 \mathrm{~d}$ gel electrophoresis followed by electrotransfer of the gel content to nitrocellulose membrane and immunoprobe for apoA-I.

The first panel is a schematic representation of HDL particles: lighter grey indicates LpA-I HDL particles (containing apoA-I without apoA-II) and darker gray indicates LpA-I:A-II HDL particles (containing both apoA-I and apoA-II) in normolipidemic control subjects. 


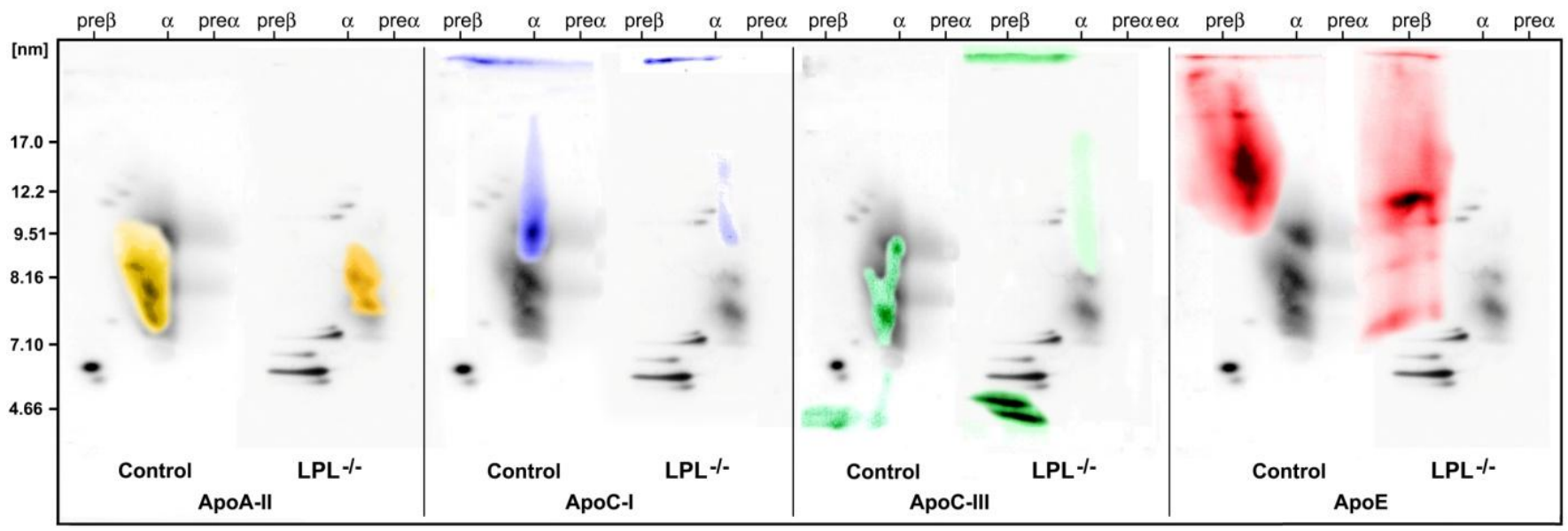

Fig. 2. ApoA-II-, apoC-I-, apoC-III-, and apoE-containing HDL subpopulations of a representative control and a homozygous LPL-deficient subject $\left(\boldsymbol{L P} \boldsymbol{L}^{-/-}\right)$. HDL particles were separated from plasma by non-denaturing $2 \mathrm{~d}$ gel electrophoresis followed by electrotransfer of the gel content to nitrocellulose membrane and immunoblot for the apolipoprotein of interest. ApoA-II- (yellow), apoC-I- (blue), apoC-III(green), and E (red) -containing HDL subpopulations are superimposed on apoA-I-containing HDL subpopulation distribution (grey). Membranes were first immunoprobed for the minor apolipoproteins and after documenting their distribution pattern, the membranes were immunoprobed for apoA-I for documenting co-migration of these apolipoproteins. 


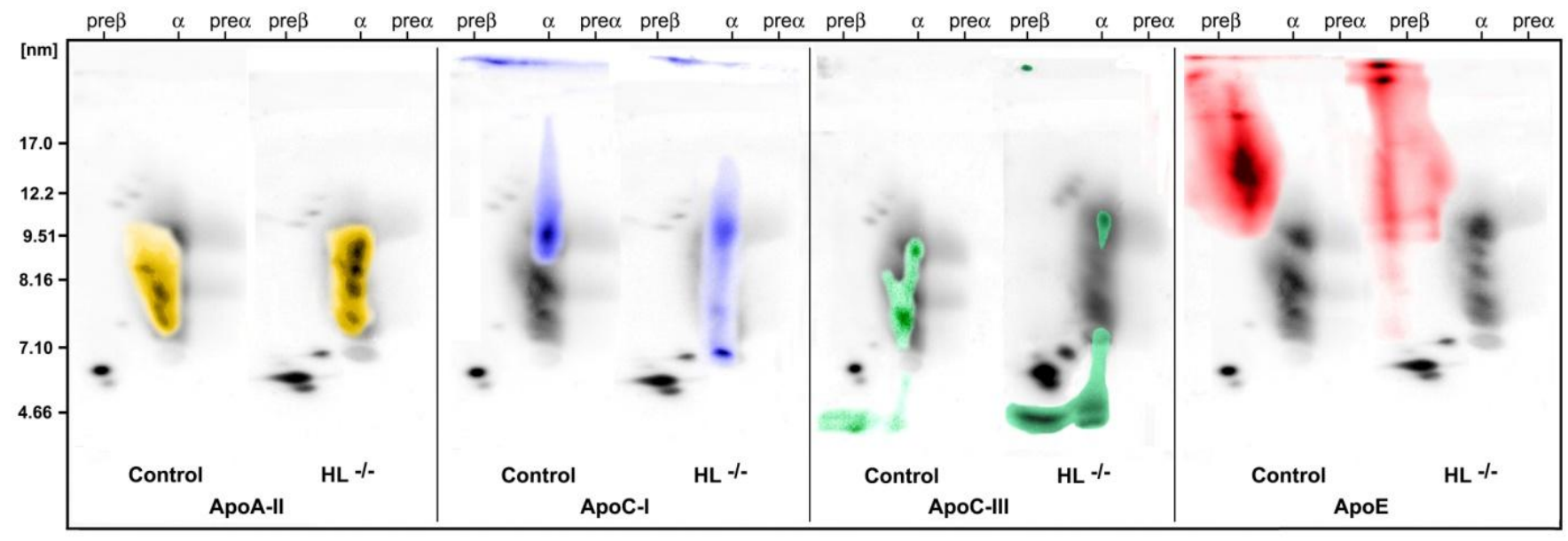

Fig. 3. ApoA-II-, apoC-I-, apoC-III-, and apoE-containing HDL subpopulations of a representative control and a homozygous HL-deficient subject $\left(\boldsymbol{H} \boldsymbol{L}^{-/}\right)$. HDL particles were separated from plasma by non-denaturing $2 \mathrm{~d}$ gel electrophoresis followed by electrotransfer of the gel content to nitrocellulose membrane and immunoblot for the apolipoprotein of interest. ApoA-II- (yellow), apoC-I- (blue), apoC-III(green), and E (red) -containing HDL subpopulations are superimposed on apoA-I-containing HDL subpopulation distribution (grey). Membranes were first immunoprobed for the minor apolipoproteins and after documenting their distribution pattern, the membranes were immunoprobed for apoA-I for documenting co-migration of these apolipoproteins. 


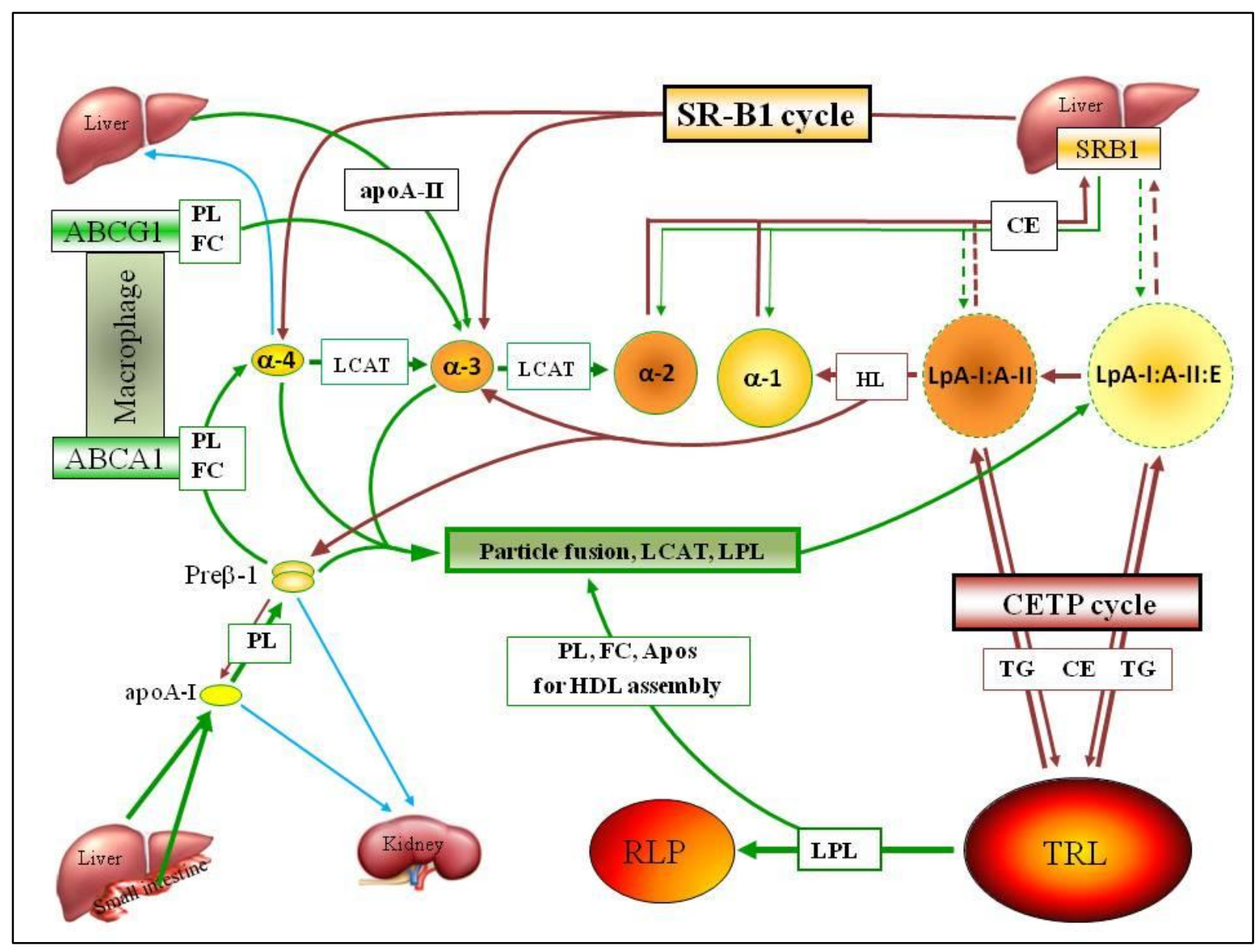

Fig. 4. A hypothetical model of the metabolism of apoA-I-containing HDL subpopulations. Green lines represent steps associated with particle maturation from small lipid-poor discs into larger more complex spherical HDL particles. Brown lines represent steps associated with HDL particle remodeling from larger into smaller particles. Blue lines represent steps associated with HDL particle catabolism. ABCA1, ATP-binding cassette transporter A1; apo, apolipoprotein; CE, cholesteryl esther; FC ,free cholesterol; HL, hepatic lipase; LCAT, lecithin:cholesterol acyltransferase; LPL, lipoprotein lipase; PL, phospholipid; RLP, remnant-like particle; SR-BI, scavenger receptor BI; TG, triglyceride; TRL. triglyceride-rich lipoproteins. 